\title{
The Gō model revisited: Native structure and the geometric coupling between local and long-range contacts
}

\author{
Patrícia F. N. Faísca, ${ }^{1, *}$ Margarida M. Telo da Gama, ${ }^{1}$ and Ana Nunes ${ }^{1}$ \\ ${ }^{1}$ Centro de Física Teórica e Computacional da Universidade de Lisboa, \\ Av. Prof. Gama Pinto 2, 1649-003 Lisboa Codex, Portugal
}

\begin{abstract}
Monte Carlo simulations show that long-range interactions play a major role in determining the folding rates of 48-mer three-dimensional lattice polymers modelled by the Gō potential. For three target structures with different native geometries we found a sharp increase in the folding time when the relative contribution of the long-range interactions to the native state's energy is decreased from $\sim 50 \%$ towards zero. However, the dispersion of the simulated folding times depends strongly on the native geometry and Gō polymers folding to one of the target structures exhibit folding times spanning three orders of magnitude. We have also found that, depending on the target geometry, a strong geometric coupling may exist between local and long-range contacts meaning that, when this coupling exists, the formation of long-range contacts is forced by the previous formation of local contacts. The absence of a strong geometric coupling leads to kinetics that are more sensitive to the interaction energy parameters; in this case the formation of local contacts is not sufficient to promote the establishment of long-range ones when these are strongly penalized energetically, leading to longer folding times.
\end{abstract}

PACS numbers: 87.15.Cc; 91.45.Ty

Keywords: lattice models, Monte Carlo simulation, native geometry, folding kinetics

\section{INTRODUCTION}

In the last few years the idea that the native geometry governs the overall folding kinetics of small (typically with less than 100 amino acids), single domain, two-state proteins has attracted considerable attention and prompted several new lines of research [1, 2, 3, 4, 5, 6, 7, 8, 9]. A particularly important observation by Plaxco et al. [1, 10] revealed the existence of a strong correlation $(r=0.92)$ between the experimental folding rates of 24 two-state folders and the so-called contact order parameter, $\mathrm{CO}$, measuring the average sequence separation of contacting residue pairs in the native structure relative to the protein chain length. The connection between the $\mathrm{CO}$ (and in more general terms, the native geometry) and the average range of amino acid interactions in the native fold has set a new ground for discussing an old-debated issue in the protein folding literature, namely, that of understanding the roles of local (i.e. close in space and in sequence) and long-range (i.e. close in space but distant in sequence) inter-residue interactions in the folding dynamics. Results obtained within the scope of this debate agree on the idea that long-range (LR) interactions play an important role in stabilizing the native fold [11, 12, 13, 14 but there is no consensus on their role in the folding kinetics. For example, very early results obtained by Gō and Taketomi 11] for a 49-residue chain on a two-dimensional square lattice suggest that local interactions accelerate both the folding and unfolding transitions. In Ref. 15] Unger and Moult have studied optimized heteropolymer sequences with chain length $N=27$ on a three-dimensional cu- bic lattice and concluded that increasing the strength of local interactions increases the ability of sequences to fold. In a different study [13], Doyle and co-workers have found that, in the context of the Zwanzig model, the rate of folding increases as the contribution of the local interactions to the native state's energy increases. By contrast, results obtained by Abkevich et al. 12 for the Miyazawa-Jernigan lattice-polymer model provided evidence that, under conditions where the native state is stable, a 36-residue sequence on a three-dimensional cubic lattice folds to a native structure with mostly LR contacts two-orders of magnitude faster than a sequence folding to a native structure with predominantly local contacts. In Ref. [16] Govindarajan and Goldstein have used a lattice model in conjunction with techniques drawn from the theory of spin glasses and found that optimal conditions for folding are achieved when local interactions contribute little to the native state's energy. More recently, Gromiha and Selvaraj have analysed the 'global' contribution of LR interactions to the folding kinetics by introducing a new geometrical parameter named longrange order (LRO) 17]. The LRO, that measures the number of LR contacts in the native structure relative to the protein chain length, was found to correlate as well as the $\mathrm{CO}$ with the folding rates of 23 (out of the 24) two-state folders previously studied by Plaxco et al [10]. This observation emphasizes the relative importance of LR interactions in protein folding kinetics.

In addition, it has been shown recently that the free energy landscapes of single domain, two-state folders are considerably smooth 22, 23. This finding led to a renewed interest [8, 27, 28] in the Go model and other modified Gō-type interaction schemes since, as for simple 
proteins, their energy landscapes are relatively smooth 28]. Indeed, these models do not take into account the sequence's chemical composition and account only for attractive interactions between native contacts thereby eliminating possible energetic traps. As a consequence only geometric traps, resulting from the chain connectivity and the geometry of the native fold, will contribute to the landscape's ruggedness and thus Gō type models are said to be frustrated in a 'topological' sense. These models are therefore particularly suited to investigate the role of the native state's geometry in the folding kinetics of simple proteins.

Motivated by these results, we revisit the Go model to investigate the dependence of the folding kinetics on LR (and local) interactions for different native geometries. Our main finding is that, for Gō-type lattice polymers with $N=48$ amino acids, the LR interactions play a crucial role in determining the folding rates and, most importantly, this effect is strongly dependent on the native state's geometry. Indeed, we have found that, depending on the native geometry, the dispersion of the simulated folding times spans up to $\approx 3$ orders of magnitude when the relative strength of $\mathrm{LR}$ interactions varies from zero (only local interactions contribute to the native state's energy) to one (only LR interactions contribute to the native state's energy). We have also found that, depending on native state's geometry, the set-up of LR contacts may be strongly associated with the previous formation of local contacts. The existence of this geometric coupling between local and LR contacts explains why the observed folding kinetics may depend rather weakly on the relative energetic contributions of local and long-range interactions. In proteins where this geometric coupling is stronger, the local contacts promote the establishment of LR contacts even when the LR interactions are not energetically favored.

The present article is organized as follows: Section II describes the model and methods. In Section III we present and discuss the results of the simulations and in Section IV we draw the conclusions.

\section{MODEL AND METHODS}

Protein chains with $N=48$ amino acids are modelled as self-avoiding walks on a three-dimensional infinite lattice. Amino acids are represented by beads of uniform size and the peptide bond, that covalently connects amino acids along the polypeptide chain, is represented by a stick of size equal to the lattice spacing. In order to mimic protein movement we use the so-called 'kink-jump' move set including corner-flips, end and null moves as well as crankshafts [24]. The Gō potential is used to model amino acid interactions which means that, for a given target native structure, equal stabilizing energies $(<0)$ are ascribed to all the native contacts, i.e. contacts between pairs of beads which are present in the target, and neutral energies $(=0)$ are ascribed to nonnative contacts, i. e., contacts that are not present in the target structure. The total energy of a conformation $\Gamma=\left\{\vec{r}_{i}\right\}$ is then given by the contact Hamiltonian

$$
H\left(\left\{\overrightarrow{r_{i}}\right\}\right)=\sum_{i>j}^{N} B_{i j} \Delta\left(\overrightarrow{r_{i}}-\overrightarrow{r_{j}}\right),
$$

where the contact function, $\Delta\left(\overrightarrow{r_{i}}-\overrightarrow{r_{j}}\right)$, is unity if beads $i$ and $j$ form a native contact but are not covalently linked and is zero otherwise and the interaction energy parameter is $B_{i j}=-\epsilon$.

The folding simulations follow the standard Monte Carlo (MC) Metropolis algorithm 25]. Each MC run starts from a randomly generated unfolded conformation (typically with less than 10 native contacts) and the folding dynamics is traced by following the evolution of the fraction of native contacts, $Q=q / Q_{\max }$, where $Q_{\max }=57$ (for chains with length $N=48$ ) and $q$ is the number of native contacts at each MC step. The folding time, $t$, is given by the first passage time (FPT), that is, the number of $\mathrm{MC}$ steps corresponding to $Q=1.0$.

\section{A. Native structures}

We consider three native structures, displaying different geometries as measured by the contact order parameter. These structures were found by homopolymer relaxation. In these $\mathrm{MC}$ simulations a homopolymeric chain (i.e., a polymer chain composed by beads of a single chemical type) is launched, at temperature $T=0.7$, from a randomly generated conformation and relaxes, after some MC steps, to the minimum energy conformation. At each MC step a local random displacement of one or two beads, provided by the kink-jump move set, is accepted or rejected in accordance with the Metropolis rule. For each conformation the total energy is given by the contact Hamiltonian of Equation $\square$ where $\Delta=1$ if beads are in contact but not covalentely linked and is zero otherwise. The pairwise interaction energy parameter is $B_{i j}=-1.0$. For homopolymers of chain length $N=48$ on a three-dimensional cubic lattice the most stable conformation, evolving under the Hamiltonian of Equation 10 is a cuboid with 57 contacts. Because this structure displays a maximum number of contacts it is generally referred to as a maximally compact structure.

In order to emphasize their different geometries, the low-CO (0.12) structure, $\Gamma_{1}$, the intermediate-CO $(0.19)$ structure, $\Gamma_{2}$, and the high-CO structure $(0.26) \Gamma_{3}$, are represented in Figures 1 (a)-(c) through their contact maps [26]. The corresponding three-dimensional structures are depicted in Figures 1(d)-(f). The contact map, $C$, is an $N \times N$ matrix with entries $C_{i j}=1$ if beads $i$ and $j$ are in contact and zero otherwise. In Ref. 17] 

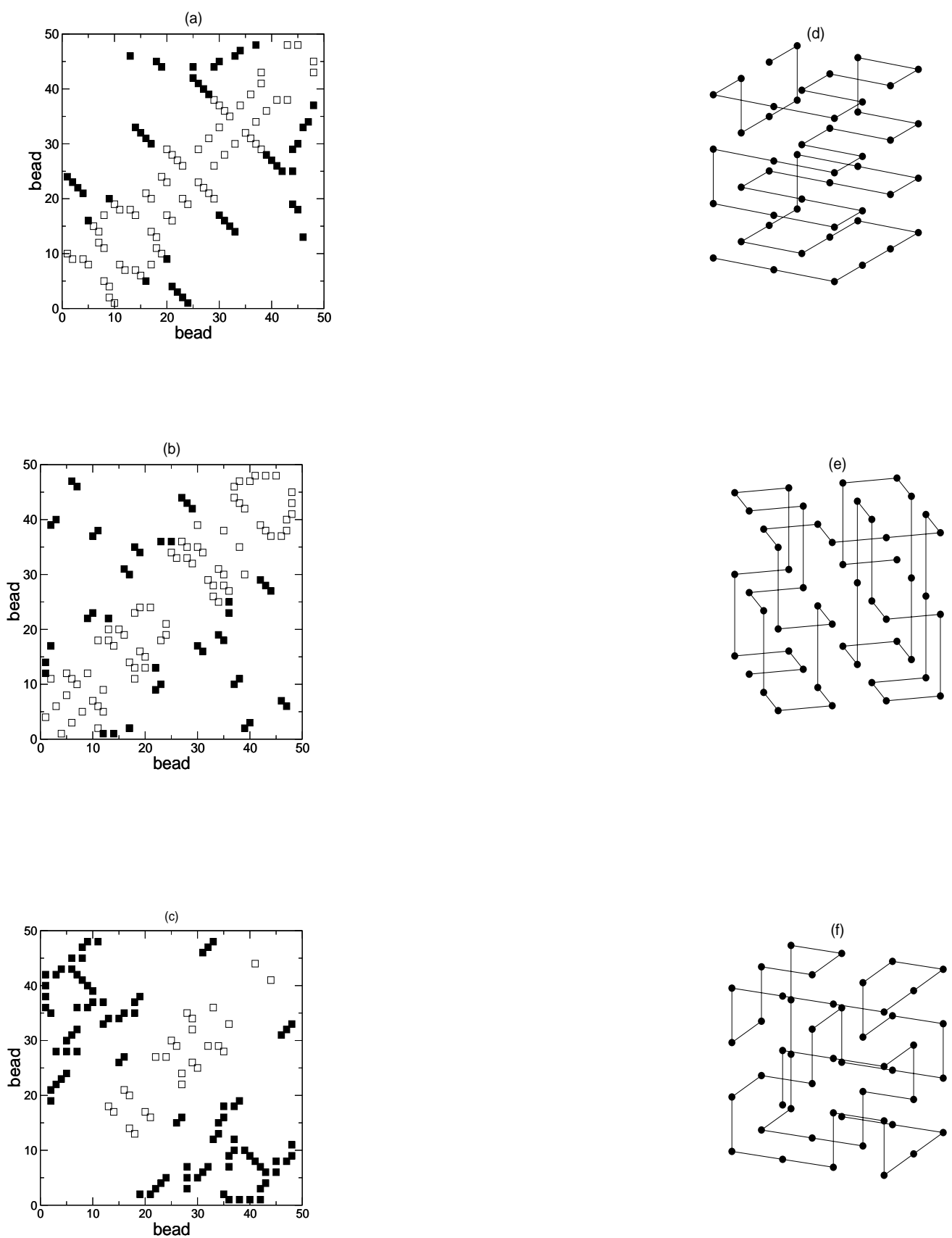

FIG. 1: Contact map and three-dimensional representation of structures $\Gamma_{1}(\mathrm{a}, \mathrm{d}), \Gamma_{2}(\mathrm{~b}, \mathrm{e})$ and $\Gamma_{3}(\mathrm{c}, \mathrm{f})$. In the contact maps the black squares represent the long-range contacts and the white squares stand for the local contacts.

Gromiha and Sevaraj have found that for real two-state proteins the amino acids which are close in space and separated by at least 10 to 15 amino acids in sequence are important determinants of folding rates. Motivated by this finding we define a native contact between two beads $i$ and $j$ as a local contact if the backbone separation $|i-j|$ is such that $|i-j| \leq 10$ or as long-range (LR) contact if $|i-j|>10$. In Figure 1 the black squares represent the LR contacts while the white squares stand for the local contacts. The LRO parameter is 0.48 for $\Gamma_{1}$, 0.44 for $\Gamma_{2}$ and 0.92 for $\Gamma_{3}$. We stress that the number of LR and local contacts is approximately the same in targets $\Gamma_{1}$ and $\Gamma_{2}$. The average LR contact length is 17.1 for $\Gamma_{1}, 20.1$ for $\Gamma_{2}$, and 26.5 for $\Gamma_{3}$. Table I summarizes the geometric traits of the three target structures. 
TABLE I: Contact order, fraction of long-range native contacts, $Q_{L R}$, long-range order and average long-range contact length for structures $\Gamma_{1}, \Gamma_{2}$ and $\Gamma_{3} . Q_{L R}$ is the number of LR native contacts normalised to the total number of native contacts.

\begin{tabular}{ccccc}
\hline \hline Target & CO & $Q_{L R}$ & LRO & $\left\langle|i-j|_{L R}\right\rangle$ \\
\hline$\Gamma_{1}$ & 0.129 & 0.40 & 0.48 & 17.1 \\
$\Gamma_{2}$ & 0.190 & 0.35 & 0.42 & 20.1 \\
$\Gamma_{3}$ & 0.259 & 0.77 & 0.92 & 26.5 \\
\hline \hline
\end{tabular}

\section{NUMERICAL RESULTS}

\section{A. Simulation temperature}

The folding kinetics depends on the temperature. Indeed, when the temperature is very high, all conformations are equally foldable and the kinetics becomes increasingly slower due to rapid interconversions (fluctuations) between unfolded states (in the high-temperature regime the folding time approaches the Levinthal time, i.e., it becomes exponential in the number of accessible conformations). On the other hand, in the lowtemperature regime, an Arrhenius-like behaviour, characterized by trapping into metastable states is expected (as discussed in [18]). Therefore, for kinetically foldable proteins, there must exist an intermediate temperature where the folding process is fastest. The existence of this temperature, called the optimal folding temperature, was reported in several studies for lattice models (sequencespecific as well as Gō models) [18, 19, 20, 21].

In the present study folding kinetics is studied at the optimal folding temperature $T^{*}$, that is, the temperature that minimises the folding time, $t$. In order to determine $T^{*}$ we performed MC simulations over a broad temperature range and ran a set of $100 \mathrm{MC}$ simulations at each temperature, $T$. The folding time was then taken as the mean FPT to the native structure averaged over the 100 $\mathrm{MC}$ runs.

Figure 2 reports the dependence of the folding time on the folding temperature for each structure and $\epsilon=0.5$. At the optimal folding temperature, the kinetics is not dominated by kinetic traps, and folding to the native state proceeds relatively fast.

We stress that, at $T \geq T^{*}$, the observed dispersion of folding times is rather small $\left(5.56 \pm 0.04 \leq \log _{10}(t) \leq\right.$ $6.11 \pm 0.05)$ and note that such behaviour is typical of the Gō and other lattice (as well as off-lattice) models (Ref. [8] and references therein.)

The functional dependence of the folding time on the temperature is qualitatively similar for the three structures in the high- $T$ regime. Note that in this regime one also observes a small dispersion of the folding times. However the reported results show that in the low- $T$ regime the dependence of the folding time on temper-

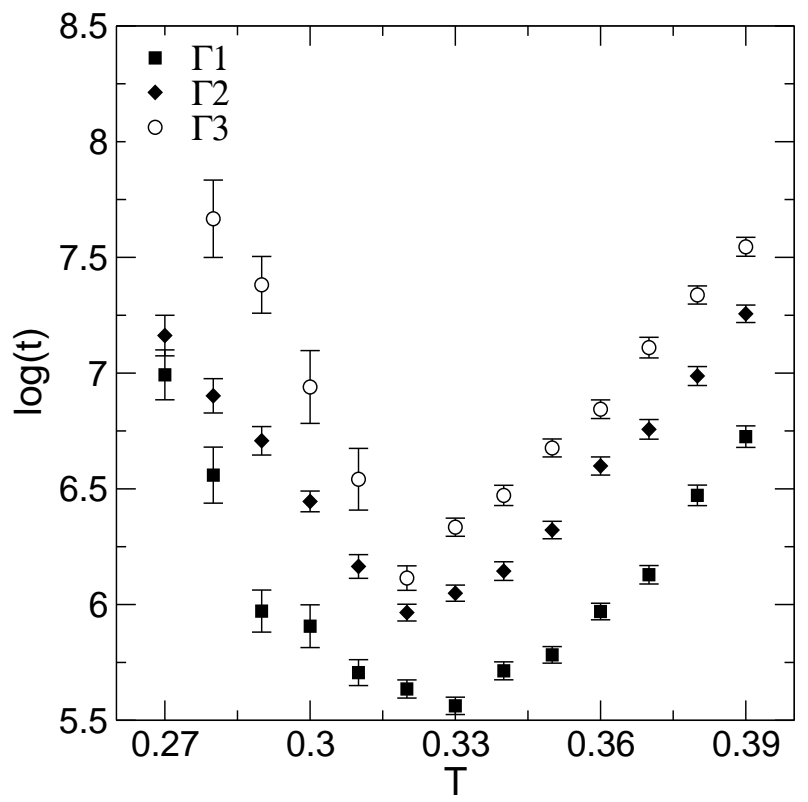

FIG. 2: Dependence of the logarithmic folding time, $\log _{10}(t)$, on the simulation temperature, $T$, for structures $\Gamma_{1}, \Gamma_{2}$ and $\Gamma_{3}$.

ature is sensitive to the native state's geometry. In particular we have not observed folding to $\Gamma_{3}$ at low temperatures, $T<0.28$.

The optimal folding temperature, on the other hand, appears to be a geometry independent parameter.

\section{B. Folding kinetics for different range bias}

In this section we investigate the role of LR and local contacts in the kinetics of protein folding by varying the relative contributions of LR and local interactions to the total energy in the following way: the energy of a conformation is given by

$$
H\left(\left\{\overrightarrow{r_{i}}\right\}\right)=\sigma H_{L R}\left(\left\{\overrightarrow{r_{i}}\right\}\right)+(1-\sigma) H_{L}\left(\left\{\overrightarrow{r_{i}}\right\}\right)
$$

where the terms $H_{L R}$ and $H_{L}$ determine the overall energy contribution of long-range and local contacts to the conformation's energy and are given by

$$
H_{L R(L)}\left(\left\{\overrightarrow{r_{i}}\right\}\right)=-\sum_{i>j}^{N} \Delta_{L R(L)}\left(\overrightarrow{r_{i}}-\overrightarrow{r_{j}}\right)
$$

where $\Delta_{L R(L)}\left(\overrightarrow{r_{i}}-\overrightarrow{r_{j}}\right)$ is unity if beads $i$ and $j$ form a $\mathrm{LR}$ (local) native contact and is zero otherwise. The parameter $\sigma$, that we shall call range bias parameter, takes values in $0.0 \leq \sigma \leq 1.0$. When $\sigma$ is zero all LR interactions are 'switched-off' and only local interactions contribute to the conformation's total energy. The reverse situation is obtained when $\sigma=1.0$ as in this case 


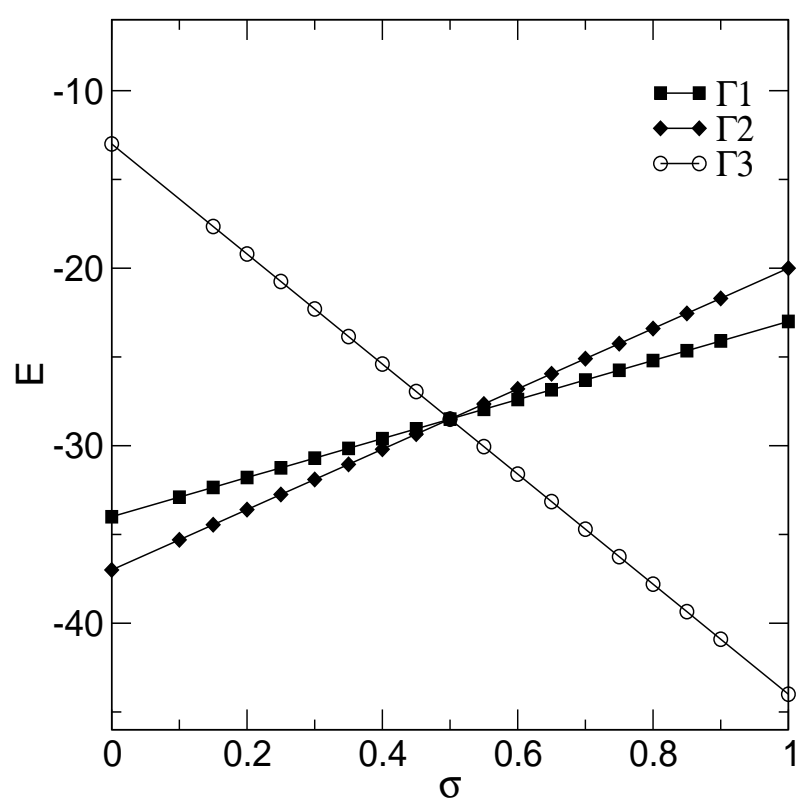

FIG. 3: Dependence of the native state's energy, $E$, on the range bias parameter $\sigma$ for structures $\Gamma_{1}, \Gamma_{2}$ and $\Gamma_{3}$.

only LR interactions contribute to the total energy. Conformation's energies given by Equation 2 imply that the native's state energy varies as a function of $\sigma$.

Results plotted in Figure 3 illustrate the dependence of the native's state energy on the range bias parameter for targets $\Gamma_{1}, \Gamma_{2}$ and $\Gamma_{3}$. While targets $\Gamma_{1}$ and $\Gamma_{2}$ have predominantly local contacts and thus their energy increases with $\sigma$ for target $\Gamma_{3}$ the lowest native state energy is observed when $\sigma=1.0$. Since the native state's energy depends on the range bias parameter we have determined, for each $\sigma$, the corresponding optimal folding temperature, $T^{*}$.

The dependence of the folding time on the range bias parameter is reported in Figure 4 (a) for the three native geometries. For $\sigma<0.20$ (resp. $\sigma<0.10$ ) we have not observed folding to target $\Gamma_{3}$ (resp. $\Gamma_{2}$ ).

The behaviour exhibited by target $\Gamma_{3}$ is easily explained: since approximately 80 percent of $\Gamma_{3}$ 's native contacts are LR there is little competition (and therefore little frustration) between LR and local interactions. We stress that, in the present model, the competition between local and LR contacts results from the relative weight of the two types of interactions (which are always stabilizing, i.e., $<0$ ). The resulting frustration is therefore different from that of sequence-specific models where the energy of the local and of the LR pair interactions can be stabilizing (i.e., $<0$ ) or de-stabilizing (i.e., $=0$ or $>0$ ). The slight decrease in the folding time observed for $\sigma>0.5$ is driven by the native state's energy (since its decrease with $\sigma$ results in a driving force to folding). However, the effect of decreasing $\sigma$ below $\sigma=0.5$ is equivalent to that of progressively 'switching-off' the LR
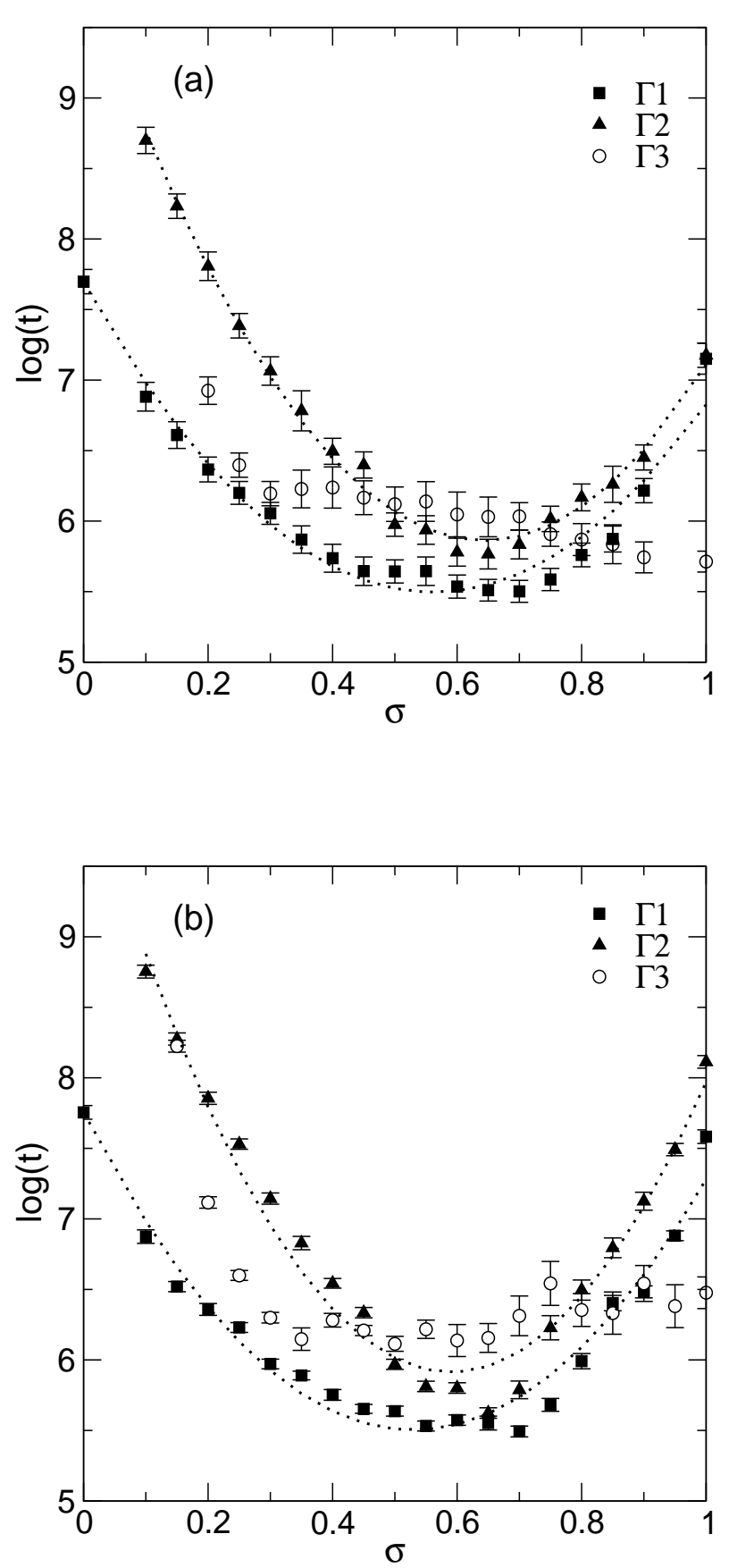

FIG. 4: (a) Dependence of the logarithmic folding time, $\log _{10}(t)$, on the range bias parameter $\sigma$ for structures $\Gamma_{1}, \Gamma_{2}$ and $\Gamma_{3}$ with different native state's energies and with a fixed native state's energy (b).

interactions and, in the limit of $\sigma=0$, it actually forces the structure to fold with only 20 per cent of its native interactions. In this case the driving force to folding decreases steadily which results in longer folding times and eventually, for $\sigma<0.20$, folding failure. The observed threshold is smaller for target $\Gamma_{2}$ because, by contrast to the behaviour of target $\Gamma_{3}$, the native state's energy decreases with $\sigma$ (for $\sigma<0.5$ ) and this effect balances 
that of switching-off the LR interactions.

The results obtained for the low- and intermediate$\mathrm{CO}$ target structures, $\Gamma_{1}$ and $\Gamma_{2}$, are more interesting. The corresponding curves are qualitatively similar but a closer inspection reveals an important difference, namely: for $\sigma<0.5$ the dependence of the folding time on $\sigma$ is much stronger for the intermediate- $\mathrm{CO}$ structure, $\Gamma_{2}$. Indeed, in this case one observes a remarkable threeorder of magnitude dispersion of folding times, ranging from $\log _{10}\left(t_{\text {min }}\right)=5.76 \pm 0.05$ (for $\sigma=0.65$ ) to $\log _{10}\left(t_{\max }\right)=8.75 \pm 0.05$ (for $\sigma=0.10$ ), by contrast with $\Gamma_{1}$ for which $\log _{10}\left(t_{\text {min }}\right)=5.50 \pm 0.08$ (for $\left.\sigma=0.70\right)$ and $\log _{10}\left(t_{\max }\right)=7.69 \pm 0.09$ (for $\sigma=0.00$ ). We note that for both structures the folding time increases considerably faster when $\sigma$ decreases than when $\sigma$ increases away from the minimum. However, in the latter case, the folding times do not deviate from each other by contrast with their behaviour for $\sigma<0.5$. We stress that for both geometries successful folding is still observed for $\sigma=1.0$; this corresponds to 'switching-off' all local interactions, which are more than half the total number of interactions in both structures.

\section{Folding kinetics for different range bias at fixed native energy}

In order to rule out differences in the folding dynamics driven by the stability of the native state we now investigate the contribution of LR and local interactions to the folding kinetics of structures $\Gamma_{1}, \Gamma_{2}$ and $\Gamma_{3}$ in the following way: the total energy of the native structure is kept fixed (and equal to $E=-28.5$ which is equivalent to taking $\epsilon=0.5$ in Equation (1) while the relative contributions of LR and local interactions are varied over the whole range. We impose the fixed energy constraint by taking the total energy given by Equation 2 and using for the long-range and local Hamiltonians

$$
H_{L R(L)}\left(\left\{\overrightarrow{r_{i}}\right\}\right)=-\sum_{i>j}^{N} \epsilon(\sigma) \Delta_{L R(L)}\left(\overrightarrow{r_{i}}-\overrightarrow{r_{j}}\right),
$$

with

$$
\epsilon(\sigma)=\frac{0.5}{(1-\sigma) Q_{L}+\sigma\left(1-Q_{L}\right)}
$$

where $Q_{L}$ is the number of local native contacts normalised to the total number of native contacts. Again, the parameter $\sigma$ determines the contribution of local and LR contacts to the total energy. For $\sigma=0.0(\sigma=1.0)$ only local (LR) contacts contribute to the total energy. However, $\epsilon(\sigma)$ that measures the interaction energy of all native contacts in $H_{L R(L)}$ varies with $\sigma$ in order to keep the total energy of the conformation constant. Using Eqs. 2 4 and [5 the energy per native contact is given by $\epsilon_{L}=(1-\sigma) \epsilon(\sigma)$ if the contact is local while it is given by $\epsilon_{L R}=\sigma \epsilon(\sigma)$ for LR contacts. Figure 5 shows the dependence of $\epsilon_{L R}$ and $\epsilon_{L}$ on the range bias parameter for structures $\Gamma_{1}, \Gamma_{2}$ and $\Gamma_{3}$.

We have studied the equilibrium population of states in order to investigate the native state's occupation probability at the optimal folding temperature as well as its dependence on $\sigma$. To this end long simulations (lasting up to $10^{8} \mathrm{MC}$ steps) were preformed in order to ensure that data was collected under equilibrium conditions [12]. The results from these simulations (for the three targets) are reported in the histograms of Figure [6] for values of $\sigma=0.3,0.5,0.7$. The height of each bar in the histograms, measures the probability occupancy, i.e., the number of molecules (normalised to the total number of molecules collected in one run) with a fraction of native contacts $Q$. In all the cases considered most molecules are in the native state $(Q=1.0)$. However, when $\sigma=0.3$, the native state of target $\Gamma_{1}$, has a rather low probability occupancy (less than 0.5). As the stability of the native state is estimated as being proportional to the probability of the chain to be in the native conformation [12], we conclude that, for $\sigma=0.3$, the native state of target $\Gamma_{1}$ is not very stable. We note that target $\Gamma_{3}$, which has the largest fraction of long-range contacts, exhibits the highest native state ocupation probability for all the three values of $\sigma$. This observation is in line with the idea that long-range contacts have a dominant role in stabilizing the native fold.

Figure 4 (b) shows the dependence of the folding time, $t$, on the range bias parameter for the three targets at fixed native state's energy. The conclusions drawn for the case of varying native state's energy hold equally well in the fixed energy case. In particular, the reported results confirm the trend for the dependence of $\Gamma_{3}$ 's folding time on the range bias parameter. We should stress, however, that in the present case folding failure is observed for $\sigma<0.15$ by contrast with the varying energy model where no successful folding was observed for $\sigma<0.20$. We ascribe this behaviour to the stabilizing (or equivalently, to the lower) native state's energy which compensates the effect of 'switching-off' the LR interactions. Hereafter we will restrict the discussion to the results for structures $\Gamma_{1}$ and $\Gamma_{2}$. We note that the main difference between the fixed and varying energy models is that for $\sigma>0.5$ the folding times are systematically longer (up to one order of magnitude for $\Gamma_{2}$ ) when the native state's energy is kept fixed. Recall from Figure 3 that when the native state's energy is allowed to vary it increases with $\sigma$ up to $E=-23$ and $E=-20$ for targets $\Gamma_{1}$ and $\Gamma_{2}$, respectively. The fixed native state's energy $E=-28.5$ is lower than the varying native energies in the range $\sigma>0.5$. Should native energy play a significant role, the folding time's dependence on $\sigma$ for $\sigma>0.5$ would be less pronounced for the fixed native energy simulations, in sharp contrast with our findings. Instead, these are consistent with the idea that the kinetics of folding is 


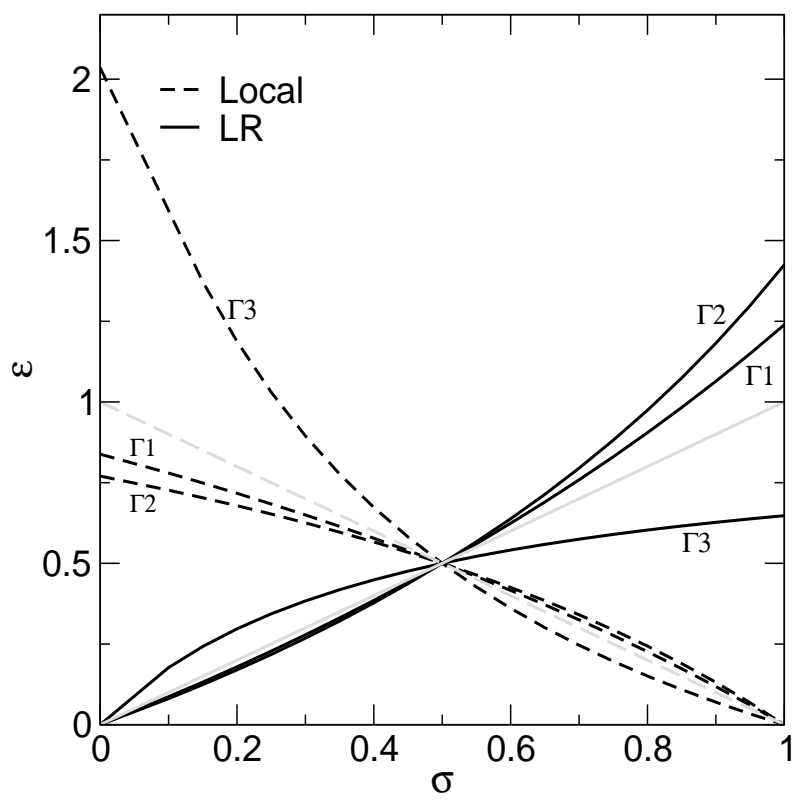

FIG. 5: Dependence of the $\epsilon_{L R}$ (energy per long range contact) and $\epsilon_{L}$ (energy per local contact) on the range bias parameter $\sigma$ for structures $\Gamma_{1}, \Gamma_{2}$ and $\Gamma_{3}$ in the fixed energy model. Also shown (in light grey) the dependence of $\epsilon_{L R}$ and $\epsilon_{L}$ on $\sigma$ in the case of the varying native energy model.

dominated by the formation of LR contacts. As shown in Figure [5 in $\Gamma_{1}$ and $\Gamma_{2}$ the energy bias favouring LR contacts for $\sigma>0.5$ is greater in the fixed energy case. This explains the differences in the behaviour of the curves corresponding to these two targets in Figures 4 (a) and प(b).

From the results reported in Figure 4 we conclude that, by comparison with local contacts, LR contacts play a crucial role, in driving the folding kinetics of small Gōtype lattice polymers. Moreover, the effect of LR contacts on the kinetics is strongly dependent on the native state's geometry.

\section{Native structure and the geometric coupling between long-range and local contacts}

In this section we investigate the differences between the folding processes of targets $\Gamma_{1}$ and $\Gamma_{2}$ when $\sigma$ is varied from zero to one in order to interpret the behaviour observed in the previous section.

Recall from section II that our 'reaction' coordinate is the fraction of native contacts $Q$. In general, $Q$ works as a thermodynamic reaction coordinate by measuring closeness to the native structure in energetic terms only. As argued in Ref. 29] thermodynamic closeness does not necessarily imply kinetic proximity to the native structure unless the energy landscape is considerably smooth [28] (as it happens to be the case in the present study). Indeed under such conditions one can take $Q$ as a kinetic reaction coordinate so that it actually defines how quickly a conformation can convert into the native structure [29]. Thus in what follows we assume that $Q$ measures the kinetic progress towards the native state.

In Figure 7 we have plotted for targets $\Gamma_{1}$ and $\Gamma_{2}$, and for three values of $\sigma$ (namely, 0.5, 0.1 and 1.0), the dependence on $Q$ of the following kinetic quantities: the fraction of LR contacts, $q_{L R}$, the fraction of local contacts, $q_{L}$ and the normalized logarithmic folding time, $\log _{10}\left(t^{*}\right)$ (note that in this case the fractions of LR and local contacts are normalized to the total number of LR and local native contacts, respectively, and not to the total number of native contacts; therefore $q=1.0$ when $Q=1.0$ but in general $q \neq Q$ and this is why the adopted notation is different from that used in the previous sections.

We start by observing the unbiased case, that is $\sigma=$ 0.5. The kinetics of local contact formation is similar in both targets with the fraction of local contacts starting from a much larger value than that of LR ones. However, long-range contacts form considerably earlier in $\Gamma_{1}$ and, in this case, the kinetics of LR contacts follows that of $q_{L}$. In both cases, the normalized folding time is controlled by the formation of local contacts. For $\sigma=1.0$ local interactions are switched-off and this results in an effective slowing-down of the corresponding kinetics in both targets. Note that, in $\Gamma_{1}, q_{L R}$ grows extremely quickly reaching $\approx 90$ per cent relatively early in the folding process (when $Q=0.58$ ) when compared with the behaviour exhibited for $\sigma=0.5$ (90 per cent when $Q=0.92$ ). However, this early boost in $q_{L R}$ does not lead to stable structure formation as it subsequently drops-down to $q_{L R}=0.84$ (for $Q=0.86$ ) and is then forced to follow the kinetics of local contact formation. For this value of $\sigma$, the folding time is controlled by the setting-up of LR contacts in $\Gamma_{2}$ and by that of local contacts in $\Gamma_{1}$. Finally, when $\sigma=0.1$, both targets exhibit a similar dependence of $q_{L}$ on $Q$ but the kinetics of $q_{L R}$ is slowed-down considerably in $\Gamma_{2}$. Again, the folding time is controlled by $q_{L}$ in $\Gamma_{1}$ and by $q_{L R}$ in $\Gamma_{2}$, but for $\Gamma_{2}$, the setting-up of LR contacts is much slower than in the previous cases.

We interpret these observations in the following way: in target $\Gamma_{1}$ there is a strong geometric coupling between the formation of local and LR contacts, meaning that the establishment of LR contacts is promoted by the establishment of local contacts. On the other hand, in target $\Gamma_{2}$, there is no such coupling and this results in an overall kinetics which is more sensitive to changes in the energy interaction parameters. In particular, in $\Gamma_{2}$, local contacts are not capable of promoting the setting-up of LR contacts when the LR interactions are highly penalized energetically (recall that we did not observe successful folding to $\Gamma_{2}$ when $\sigma<0.10$ ). 


\section{CONCLUSIONS AND FINAL REMARKS}

In this paper we have revisited the Gō model in order to investigate, by means of a new approach, the role of long-range(LR) and local interactions in the folding kinetics. We have focused our analysis on lattice-polymers with chain length $N=48$ since, like real two-state folders, these models exhibit relatively smooth energy landscapes. We studied the changes in the folding process induced by unbalancing the contributions of local and LR interactions to the native state's energy. Our results strongly suggest that LR interactions play a dominant role in the folding kinetics. Indeed we observe a decrease in the folding rates, or equivalently, an increase in the folding time, which is clearly more pronounced when the contribution of the LR interactions (relative to that of the local interactions) to the native state's energy is progressively decreased towards zero. We have found that this effect is essentially independent of the native state's energy. By contrast, the kinetic response to decreasing the relative contribution of the LR interactions is strongly dependent on the target geometry. We have selected our target geometries on the basis of differing contact order parameters. In the high-CO target, $\Gamma_{3}$, LR contacts are the vast majority (44 out of 57 native contacts) and this results in a trivial kinetic response: when LR interactions are strengthened relative to the local ones there are no significant changes in the folding rates; on the other hand, a strong increase in the folding rates (eventually resulting in folding failure) arises when they are weakened. Interesting behaviour occurs in the folding kinetics of the other two structures, the low- and intermediate-CO targets, $\Gamma_{1}$ and $\Gamma_{2}$, respectively. In both structures local contacts dominate and both exhibit a similar fraction of local and LR contacts. However, in the intermediate-CO target the kinetics is much more sensitive to the weakening of LR interactions; in fact in this case we observed a remarkable three-order of magnitude dispersion of folding times, although this is still two-orders of magnitude smaller than the dispersion of folding times of real twostate folders ( $\approx 5$ orders of magnitude) [10].

The topomer search model (TSM) for protein folding (reviewed in 31]) considers that the folding time is determined by the difusive search for the ensemble of unfolded structures that share a similar, global topology with the native state (the native topomers) 32]. Achieving the native topomer corresponds to surmounting the rate limiting step in folding, which is followed by the zippering of specific local native contacts, a process that rapidly leads to the native structure. Thus, according to the TSM, the rate at which an unfolded protein diffuses between distinct topologies is much slower than the rate at which local structural elements form. Recent results obtained through numerical simulations of the diffusion of Gaussian chains by Makarov et al. [31, 33] suggest, in the con- text of this model, that the logarithmic folding rate grows linearly with the number $N_{L R}$ of LR contact pairs in the native structure, which define the topomer. Makarov et al. 31 investigated wether the TSM correlates well with the folding rates of the 24 two-state folders previously studied by Plaxco et al. 10]. To determine $N_{L R}$ for each protein the authors have considered as LR a native contact where the amino acids are separated by at least 12 or more residues along the protein backbone. A considerably strong correlation $(r=0.88)$ was found between the logarithmic folding rates and $N_{L R}$ 31], suggesting that the TSM is indeed a plausible model for two-state folding rates. Our results are in broad agreement with the TSM in the sense that, irrespectively of target geometry, we have found that decreasing, versus increasing, the relative weigth of $\mathrm{LR}$ interactions leads to a more pronounced increase of the folding times. However, we have also found evidence for a folding mechanism (on the lattice) that is different from that of the TSM. According to the TSM, the step that determines the folding rates is the formation of the LR contacts in the native topomer. After this step a rapid zippering of the local contacts occurs and the native structure forms. Our results show that, depending on native geometry, the formation of LR contacts may be more strongly coupled with the formation of the local contacts. This is clearly illustrated by the behaviour of target $\Gamma_{1}$ when $\sigma=0.1$ (Figure [7). For this value of $\sigma$ the LR contacts are strongly penalized energetically, and the folding time is controlled by local contact formation. This is not observed for target $\Gamma_{2}$, where under the same conditions $(\sigma=0.1)$ the folding time is controlled by LR contact formation (Figure 7), in agreement with the TSM. Another result that supports the existence of coupling between local and LR contact formation in target $\Gamma_{1}$ is the fact that, again for $\sigma=0.1$, LR contact formation is much faster for target $\Gamma_{1}$ than for target $\Gamma_{2}$. We note that this particular aspect of the folding process in lattice models has not been explored in previous simulation efforts.

In a recent study Micheletti et al. 34] have introduce a novel method, the so-called 'geometrical variational principle', to investigate the role of native geometry in guiding the protein to the native fold. This study consisted in computing the number of structures that share a certain structural similarity with a given native structure (the structural similarity between a structure and the fixed native fold is measured by the fraction of native contacts $Q$ in that structure). The authors have called this measure the density of overlapping conformations (DOC). A crucial result from this study was the finding that the DOC of real natural folds is always much larger (at any value of $Q$ ) than that found in artificially generated structures (with the same chain length and number of contacts but differing in the fractions of local and non-local contacts). Moreover, the authors found that, for $Q \approx 0.5$, the DOC of real folds is very close to its 
maximum value and that this 'extremality' of the DCO is related with a high content in secondary-structure-like motifs (alpha-helices and beta-sheets). In a subsequent study Maritan el al. 35] applied a 'dynamical variational principle' (DVP) to search for rapid folders in conformational space. The authors have found, in the context of a Go model on a fcc lattice, that decreasing folding times are associated with increasing secondary structure content (in agreement with Micheletti's results) and with decreasing contact order. This finding shows that the aplication of the DVP to search for kinetically foldable proteins results in the emergence of structures with predominantly helical order (i.e., with a high content in local contacts). Since our results were obtained for a cubic lattice a detailed comparison with Maritan's findings is not possible. However, in the contact map of Figure 1(a), corresponding to $\Gamma_{1}$, one can clearly identify a pattern that resembles that of alpha-helices namely, the existence of thick bands parallel to the main diagonal. The fact that $\Gamma_{1}$ exhibits the shortest folding times for all values of the LR interaction strength is in broad agreement with Maritan's results.

The existence of a geometric coupling between local and LR contacts may have implications in what concerns the understanding of protein evolution in the sense that it provides a possible mechanism for the emergence of mutational robustness in proteins. A biological system is said to be robust to mutations if it continues to function after genetic changes in any of its parts. Native structures endowed with a mechanism of local-LR contact coupling are naturally more capable of exhibiting a fast adaptation to mechanisms of biomolecular variation (point mutations, insertions, deletions, etc) that change the amino acid sequence (i.e. that change the set of amino acid interactions) in the following sense. If the geometric coupling between local and LR contacts exists, one expects the protein's foldability (the protein's ability to fold at a reasonably fast rate which is indeed an evolutionary advantage) to be less affected by changes in the way the amino acids interact since when the LR contacts become energetically penalized, as a result of sequence changes, the establishment of local contacts acts as a driving force for the establisment of LR ones.

Finally, it would be interesting to investigate the interplay between target geometry and favored native contact interactions in more realistic models, where not only the dispersion of folding times of real proteins is reproduced as well as other aspects observed in the folding of real two-state folders such as the thermodynamic and the kinetic cooperativities [30]. A simple model that is a step in this diection is that of Kaya and Chan who used a modified Gō-type potential, involving nonadditive multybody interactions, to study the folding dynamics of 27 mers on a cubic lattice [8]. When applied to a pool of targets comprising 97 native geometries, chosen on the basis of their CO parameters, Kaya and Chan's model yielded folding rates spanning more than 2.5 orders of magnitude. Furthermore this model also exhibited thermodynamic cooperativity and linear chevron plots (i.e., kinetic cooperativity) similar to those observed in experiments with real proteins.

* Correspondence to PFN Faísca, Centro de Física Teórica e Computacional da Universidade de Lisboa, Av. Prof. Gama Pinto 2, 1649-003 Lisboa Codex, Portugal E-mail:patnev@alf1.cii.fc.ul.pt

\section{REFERENCES}

[1] Plaxco KW, Simmons KT, Baker D. Contact order, transition state placement and the refolding rates of single domain proteins. J Mol Biol 1998;277:985-994.

[2] Du R, Pande VS, Grosberg AY, Tanaka T, Shakhanovich E. On the role of conformational geometry in protein folding. J Chem Phys 1999;111:10375-13080.

[3] Baker D. A surprinsing simplicity to protein folding. Nature (London) 2000;405:39-42.

[4] Dokholyan NV, Li L, Ding F, Shakhnovich EI, Topological determinants of protein folding. Proc Natl Acad Sci USA 2002;99:8637-8641.

[5] Miller EJ, Fischer KE, Marqusee S. Experimental evaluation of topological parameters determining proteinfolding rates. Proc Natl Acad Sci USA 2002;99:1305910363.

[6] Faisca PFN, Ball RC. Topological complexity, contact order and protein folding rates, J Chem Phys 2002; 117:8587-8591.

[7] Weikl TR, Dill KA. Folding rates and low entropy routes of two-state proteins. J Mol Biol 2003;329:585-598.

[8] Kaya H, Chan HS, Contact order dependent protein folding rates: Kinetics consequences of a cooperative interplay between favourable nonlocal interactions and local conformational preferences. Proteins 2003;52:524:533.

[9] Faisca PFN, Telo da Gama MM, Ball RC. Folding and form: Insights from lattice simulations. Phys Rev $\mathrm{E}$ 2004;69:051917.

[10] Plaxco KW, Simmons KT, Ruczinski I, Baker D. Topology, stability, sequence and length: Defining the determinants of two-state protein folding kinetics. Biochemistry 2000;39:11177:11183.

[11] Gō N, Taketomi H. Respective roles of short- and longrange interactions in protein folding. Proc Natl Acad Sci USA 1978;75:559-563.

[12] Abkevich VI, Gutin AM, Shakhnovich EI. Impact of local and non-local interactions on thermodynamics and kinetics of protein folding. J Mol Biol 1995;252:460-471

[13] Doyle R, Simons K, Qian H, Baker D. Local interactions and the optimization of protein folding. Proteins 1997;29:282-291.

[14] Gromiha MM and Selvaraj S. Importance of long-range interactions in protein folding. Biophys Chem 1997;77:4968.

[15] Unger E, Moult J. Local interactions dominate folding in a simple protein model. J Mol Biol 1996;259:988-994. 
[16] Govindarajan S, Goldstein RA. Optimal local propensities for model proteins. Proteins 1995;22:413-418.

[17] Gromiha MM, Selvaraj S. Comparison between longrange interactions and contact order in determining the folding rate of two-state folders: Application of long-range order to folding rate prediction. J Mol Biol 2001;310:27-32.

[18] Gutin AM, Abkevich VI, Shakhnovich, EI. Chain length scaling of protein folding. Phys Rev Lett 1996;77:54335436.

[19] Gutin A, Sali A, Abkevich V, Karplus M, Shakhnovich EI. Temperature dependence of the folding rate in a simple protein model: Search for a glass transition. J Chem Phys 1998:108;6466-6483.

[20] Scaling of folding properties in simple models of proteins. Phys Rev Lett 1999;83:1684-1687.

[21] Faisca, PFN, Ball RC. Thermodynamic control and dynamical regimes in protein folding. J Chem Phys 2002;116:7231-7238.

[22] Gillespie B, Plaxco KW. Nonglassy kinetics in the folding of a simple single-domain protein. Proc Natl Acad Sci USA 2000;97:12014-12019.

[23] Shea JE, Onuchic JN, Brooks CL. Probing the folding free energy landscape of the src-SH3 protein domain. Proc Natl Acad Sci USA 2002;99:16064-16068.

[24] Landau DP, Binder K. A Guide to Monte Carlo Simulations in Statistical Physics. Cambridge:Cambridge University Press;2000.122p.

[25] Metropolis N, Rosenbluth AW, Rosenbluth MN, Teller AH, Teller E. Equation of state calculations by fast computing machines. J Chem Phys 1953;21:1087-1092.

[26] Saitoh S, Nakai T, Nishikawa K. A geometric constraint approach for reproducing the native backbone conforma- tion of a protein. Proteins 1993;15:191-204.

[27] Fan K, Wang J, Wang W. Modeling two-state cooperativity in protein folding. Phys Rev E 2001;041907.

[28] Jewett AI, Pande VS, Plaxco KW. Cooperativity, smooth energy landscapes and the origins of topology-dependent protein folding rates. J Mol Biol 2003;326:247-253.

[29] Chan HS, Dill KA. Protein folding in the landscape perspective: Chevron plots and Non-Arrhenius kinetics. Proteins 1998;30:2-33.

[30] Chan HS, Shimizu S, Kaya H. Cooperativity Principles in Protein Folding. Methods in Enzymology 2004;380:350379.

[31] Makarov DE, Plaxco KW. The topomer search model: A simple, quantitative theory of two-state protein folding kinetics. Prot Sci 2003;12:17-26.

[32] Debe DA, Carlson MJ, Goddard WA. The topomersampling model of protein folding. Proc Natl Acad Sci USA 1999;96:2596-2601.

[33] Makarov DE, Metiu H. A model for the kinetics of protein folding: Kinetic Monte Carlo simulations and analytical results. J Chem Phys 2002;116:5205-5216.

[34] Maritan A, Micheletti C, Banavar, JR. Role of secondary motifs in fast folding polymers: A dynamical variational principle. Phys Rev Lett 2000;3009-3012.

[35] Micheletti C, Banavar JR, Maritan A, Seno F. Protein structures and optimal folding from a geometrical principle. Phys Rev Lett 1999;3372-3375.

This is a preprint of an article accepted for publication in Proteins: Structure, Function and Bioinformatics. Copyright(1999-2005) John Wiley \& Sons. All rights reserved 

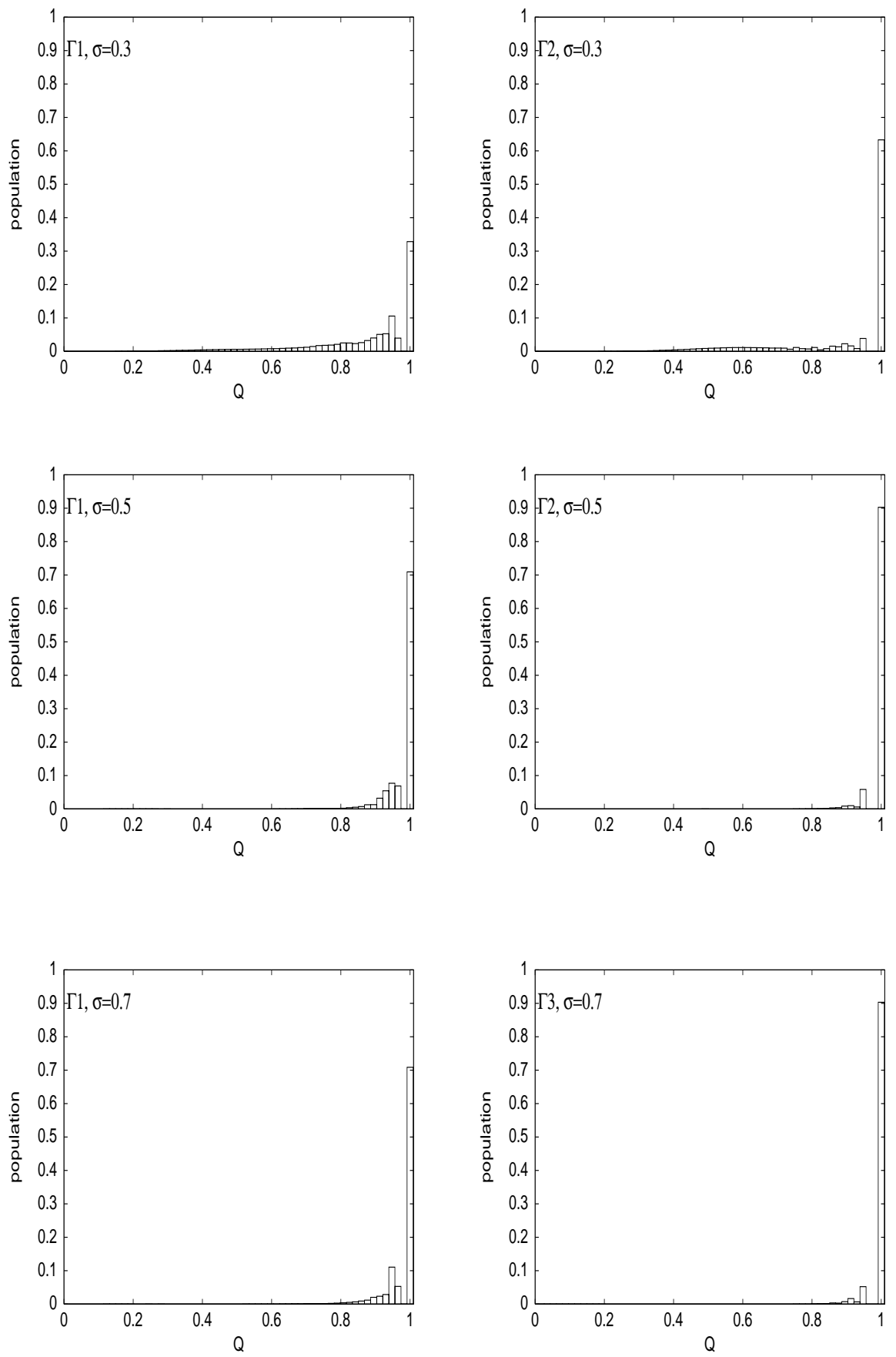

FIG. 6: Population histograms, for targets $\Gamma_{1}$ (first row), $\Gamma_{2}$ (second row) and $\Gamma_{3}$ (third row) and $\sigma=0.3,0.5$ and 0.7 at the optimal folding temperature. The native state, corresponding to $Q=1.0$, is the dominant state for all structures at all values of $\sigma$. Except for structure $\Gamma_{1}$ at $\sigma=0.3$ the native state's occupation probability is larger than 0.5 . Target $\Gamma_{3}$, with the largest fraction of long-range contacts, exhibits the largest native state ocupation probability at all values of $\sigma$. This observation is in line with the idea that long-range contacts have a dominant role in stabilizing the native fold. 

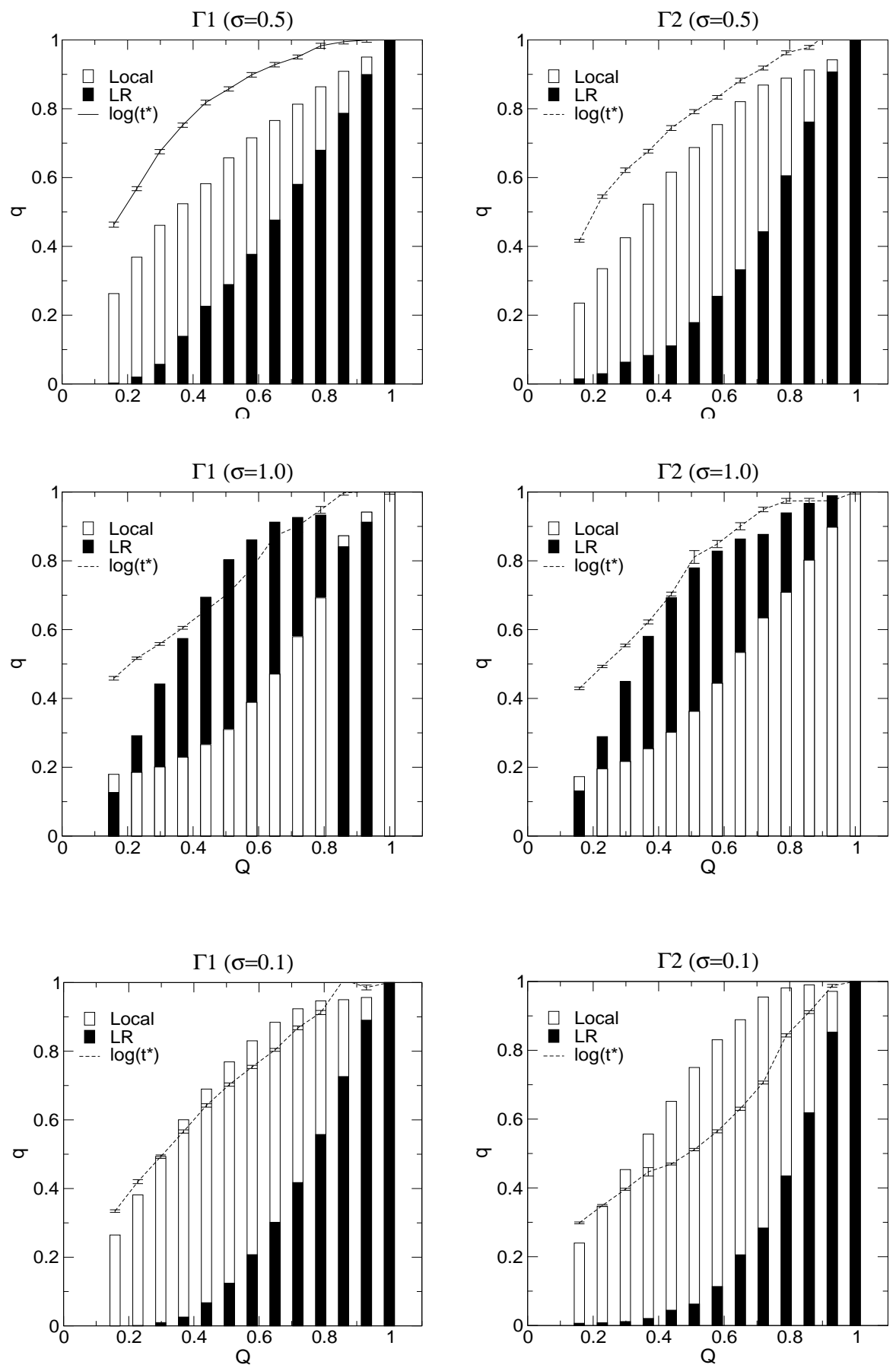

FIG. 7: Dependence of the fractions of long-range (LR) and local contacts on the fraction of native contacts, $Q$, for targets $\Gamma_{1}$ and $\Gamma_{2}$, and $\sigma=0.5,1.0$ and 0.1. Note that $q$ is $q_{L R}$ (i.e., the fraction of LR contacts) for the black bars and $q_{L}$ (i.e., the fraction of local contacts) for the white bars. $q_{L R}\left(q_{L}\right)$ is the number of LR (local) contacts normalised to the total number of LR (local) contacts in each native structure. Also shown is the dependence of $\log _{10}\left(t^{*}\right)$ on $Q$ where $t^{*}$ is folding time at each $Q$ normalised to the folding time at $Q=1.0$. 


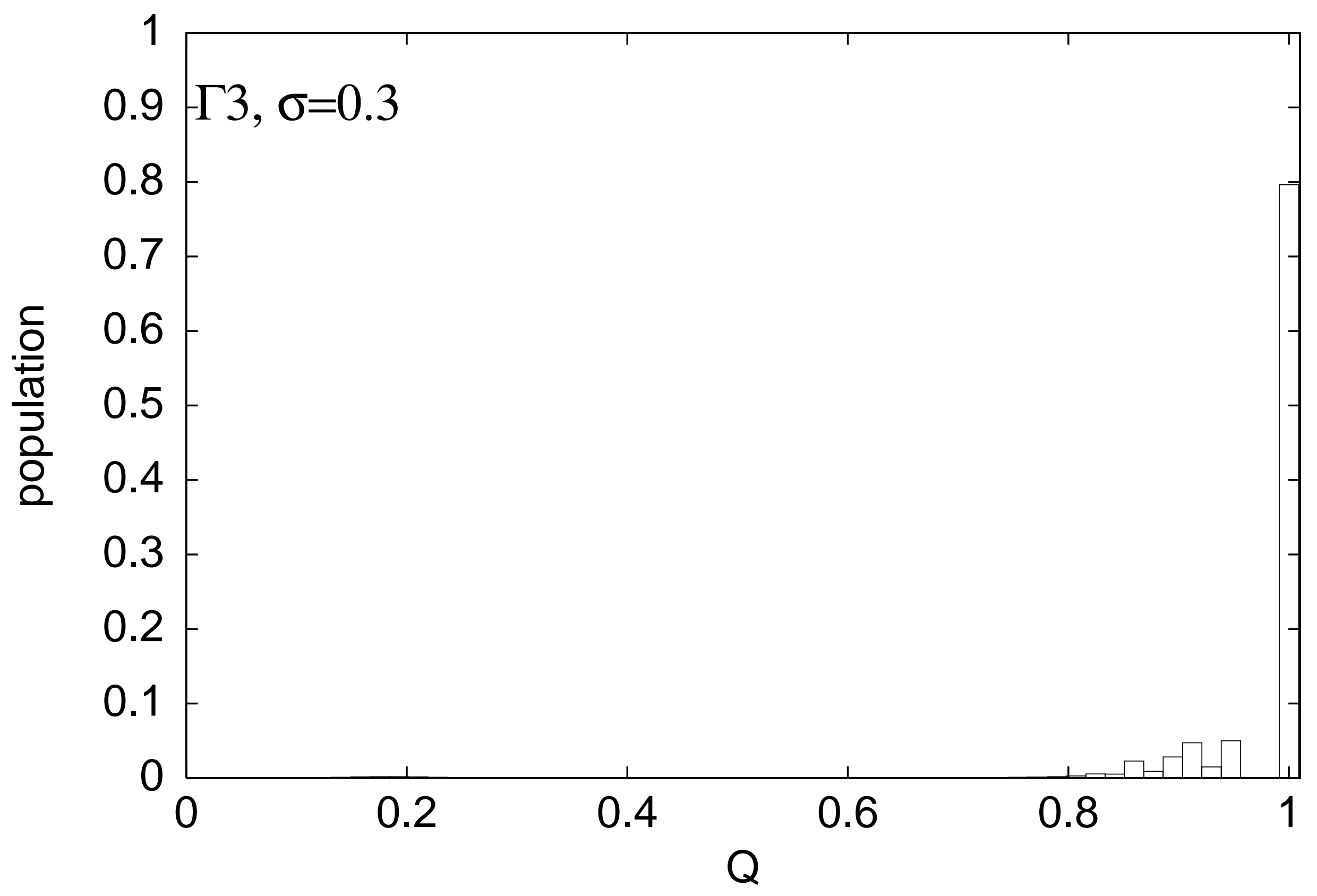




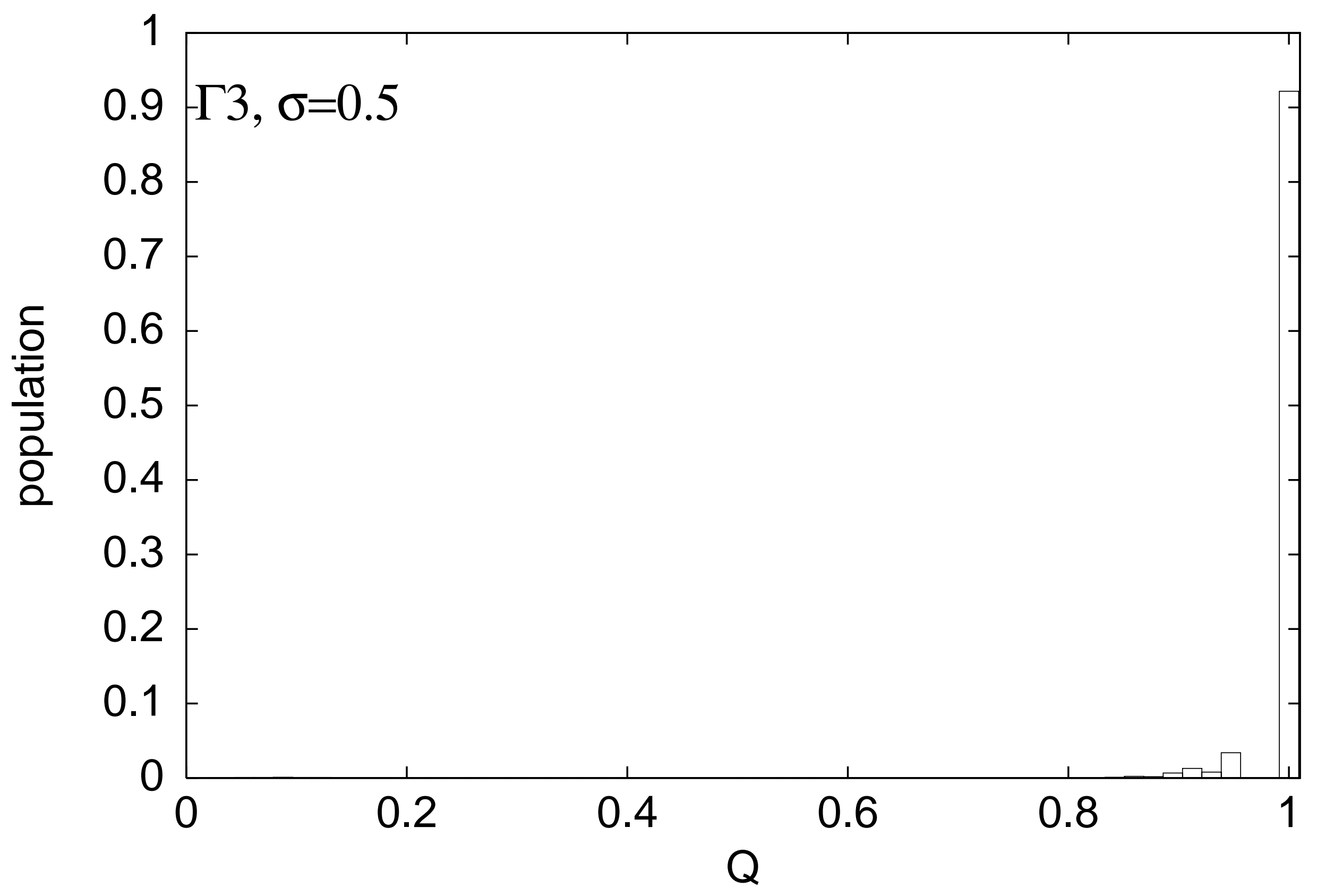




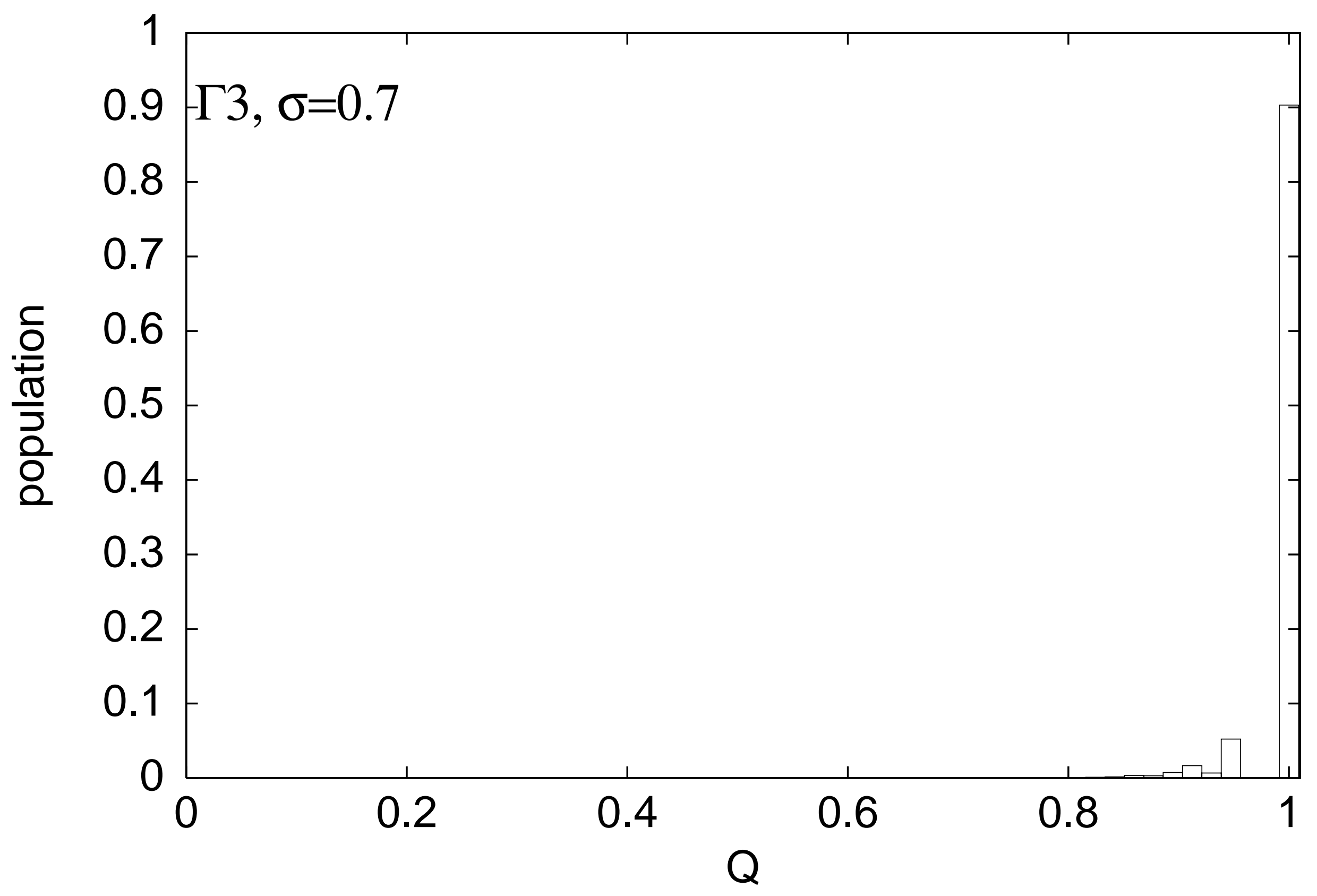

